\title{
Spatial memory and perspective taking
}

\author{
AMY L. SHELTON \\ Johns Hopkins University, Baltimore, Maryland \\ and \\ TIMOTHY P. MCNAMARA \\ Vanderbilt University, Nashville, Tennessee
}

\begin{abstract}
Giving directions or describing an environment often requires assuming perspectives other than one's own. We employed a spatial perspective-taking task to investigate how describing familiar versus novel perspectives affects subsequent memory. One participant (the director) viewed a display of objects from a single perspective and described the display to another participant (the matcher) from a perspective that varied by $0^{\circ}, 45^{\circ}, 90^{\circ}, 135^{\circ}$, or $180^{\circ}$ from the viewing perspective. Following the description, we assessed the director's memory for the display, using judgments of relative direction, scene recognition, and map drawing. The participants imagined and recognized familiar views faster and/or more accurately than novel views. Moreover, different tasks showed different degrees of facilitation for the visually perceived and described views, suggesting multiple representations for different aspects of spatial memory. These findings emphasize the importance of understanding distinctions among spatial experiences and underscore differences in the tasks used to probe spatial memory.
\end{abstract}

When navigating the complex environments of everyday life, we often rely on memories of the spatial relations among objects and the features they contain. For example, to get from the parking lot to the office each day, we must use memory for the locations of each of these places to select a path. With few exceptions, we can find our way in familiar environments with seemingly little conscious effort. In addition, we can use our memory for familiar environments to describe the spatial layout to others. For example, imagine that a graduate student is sitting at a computer in the lab and a research assistant in the same room is looking for a stopwatch. The graduate student might instruct the researcher to go to a particular desk in the lab, and then, without moving from her location, she might say, "Just to your right there are three drawers; the stopwatch should be in the middle drawer near the front left." In this scenario, the graduate student is sitting in one perspective and trying to take on the perspective of the research assistant sitting at the desk. The graduate student may be describing a familiar perspective (e.g., she was sitting at the desk when she put the stopwatch away), or she may be inferring a novel perspective from the visual scene to make finding the stopwatch easier for the research assistant. The present study was designed to address how providing spatial descriptions of familiar and

The research reported in this article was supported in part by $\mathrm{Na}-$ tional Institute of Mental Health Grants MH57868 and MH12638. We thank Thomas Palmeri, Jeffrey Schall, John Rieser, Jon Holbrook, Holly Taylor, and two anonymous reviewers for their comments on the research. Correspondence concerning this article should be addressed to A. L. Shelton, Department of Psychological and Brain Sciences, Johns Hopkins University, Ames Hall, 3400 N. Charles Street, Baltimore, MD 21218 (e-mail: ashelton@jhu.edu). novel perspectives informs us about and participates in the way space is represented in the brain.

Research on spatial descriptions has suggested that people use different types of locative statements. In all locatives, location is specified relative to some placeholder in space (the observer, another person or object, etc.). In addition, the language used to specify the location can be absolute (e.g., north of ) or relative to another position and orientation (e.g., left of ). For example, in the description above, "just to your right" reflects a relative reference with the observer as the referent, whereas "near the front left" is a relative reference with the intrinsic axes of the drawer as the referent. Alternatively, if the speaker had said, "Look in the drawers at the north end of the lab," this would be an absolute locative with the lab walls as the referent. For the present study, the key issue is whether understanding the locative requires an understanding of the observer's position and orientation in space (e.g., right or left relative to the observer) or another object's (or observer's) position and orientation in space (e.g., right or left relative to some object's intrinsic axes). These two types of locatives can be described as relative and intrinsic, respectively (Levinson, 1996). Within the intrinsic locatives, we can distinguish those that are based on the axes of another observer, the axes of an object, or properties of the environment.

Studies of spatial descriptions can reveal preferences for certain types of reference frames. For example, when people were asked to describe their apartments, they tended to take the listener on a mental tour, describing a path through the apartment in the second person (Linde \& Labov, 1975). This description is much like the route descriptions used to study route versus survey learning (Shelton \& McNamara, 2004; Taylor \& Tversky, 1992). 
Similarly, when describing the location of furniture in a dollhouse, people tended to assume an egocentric position outside the space (Ehrich \& Koster, 1983); that is, they assume a gaze perspective (Taylor \& Tversky, 1996). In the examples above, the reference frame of the description is relative; that is, locations are specified in reference to the observer's location. However, Taylor and Tversky (1996) have pointed out that people use a wide variety of locatives in normal descriptions, including relative locatives (on my left) and neutral absolute locatives (in the southwest corner). These disparate findings suggest that different locatives may be appropriate for different spaces and raise important questions about what perspective taking and spatial descriptions reflect about the underlying representations.

Work from the domain of communication may provide some initial clues. Spatial descriptions have been used to examine the various social roles that people assume, placing particular emphasis on which party's effort is minimized in conversations (e.g., Clark \& Wilkes-Gibbs, 1986; Isaacs \& Clark, 1987; Schober, 1993, 1995). The use of the spatial domain has been prominent in this literature because of the ease with which egocentric orien- tation can be identified in a space. The intent of researchers who have investigated perspective taking has not been to understand the underlying spatial representations involved; however, in using the spatial domain, these investigators have laid some groundwork for using perspective taking as a measure of spatial memory representations. Perspective taking provides a situation in which people have clearly demonstrated the ability to assume perspectives other than their own; it therefore allows us to observe the consequences associated with doing so.

Schober (1995) has provided one of the most direct examinations of the role of egocentric orientation in conversations. He used large "pizza" displays consisting of sets of 24 shapes scattered on a tabletop disk (see Figure 1). The task required that one participant, the director, describe a display to another participant, the matcher. The director and the matcher were each placed in front of identical displays so that they were facing the display from the same orientation $\left(0^{\circ}\right.$ offset $)$ or different orientations $\left(90^{\circ}\right.$ or $180^{\circ}$ offset). Figure 1 shows a sample display from the director's orientation, with the matcher's orientation indicated for the three offset conditions. Chips numbered from 1 to 18 were placed on some of the shapes in the director's

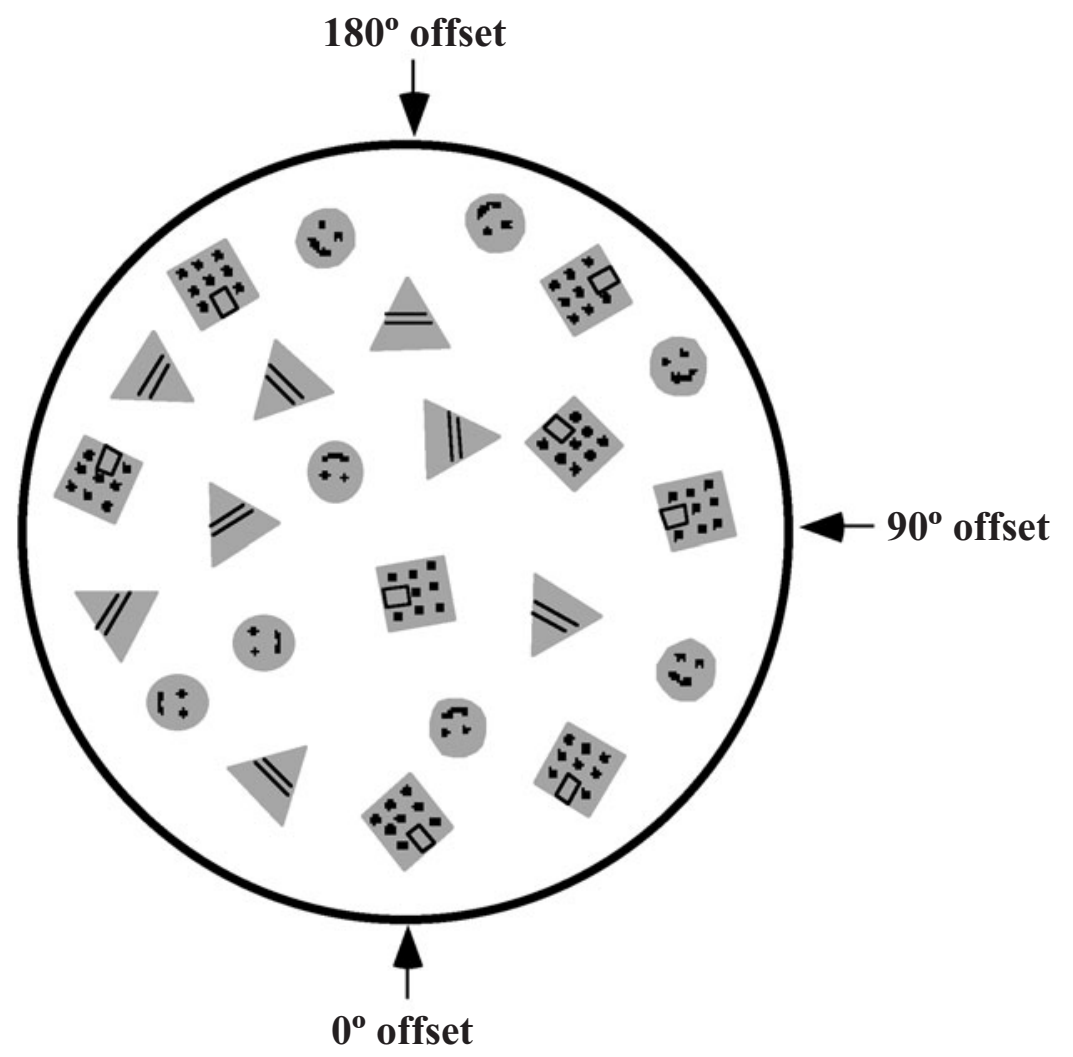

Figure 1. Example of the stimuli used by Schober (1995). Upright represents the director's perspective, and arrows indicate the perspective of the matcher in the $0^{\circ}, 90^{\circ}$, and $180^{\circ}$ offset conditions. From "Speakers, Addresses, and Frames of Reference: Whose Effort Is Minimized in Conversations About Locations?" by M. F. Schober, 1995, Discourse Processes, 20, p. 231. Copyright 1995 by Lawrence Erlbaum Associates. Adapted with permission. 
display, and the task required the director to instruct the matcher to place identical chips on the correct locations in the matcher's display.

The conversation between the director and the matcher was recorded and transcribed. Each statement in the conversation was coded as belonging to one of five perspectives: director centered (relative), matcher centered (locally intrinsic), both centered (relative, $0^{\circ}$ offset only), object centered (locally intrinsic), and neutral, environment centered (globally intrinsic). In the results of primary interest, the $0^{\circ}$ offset was compared with the $90^{\circ}$ and $180^{\circ}$ offsets. At $0^{\circ}$ offset, where the director and the matcher shared the same orientation, there was a predominant use of both-centered locatives. At $90^{\circ}$ and $180^{\circ}$ offsets, where the director and matcher had different orientations, there was an increase in matcher-centered and neutral locatives, with the neutral locatives dominating. Both the director and the matcher knew of each other's location relative to the display. As such, the director could have continued to use director-centered locatives; however, the choice to switch to neutral locatives is consistent with previous work suggesting that directors tend to minimize the effort for the matcher in conversations (e.g., Clark \& Wilkes-Gibbs, 1986; Garrod \& Anderson, 1987).

On the basis of this minimization argument, we can make certain assumptions about locatives and the effort they require in perspective taking. First, the dominance of the both-centered locatives in the $0^{\circ}$ offset conditions suggests that using locatives relative to an observer may be preferable to using intrinsic locatives. For the non- $0^{\circ}$ offsets, it is believed that the directors chose to minimize the matcher's effort, thereby increasing his or her own effort. The evidence for this is the absence of directorcentered locatives in the non $-0^{\circ}$ offset conditions. The selection of neutral locatives over matcher-centered locatives for these non- $0^{\circ}$ offsets suggests that actually switching to the matcher's perspective was more effortful than using the globally intrinsic spatial language of the neutral locatives. By this argument, the self-centered, relative locatives required the least effort, and neutral, globally intrinsic locatives required less effort than did the locally intrinsic locatives (relative to another observer's egocentric orientation).

One interpretation of the added "effort" involved in perspective switching is that the new perspective required to specify a matcher-centered locative is at odds with the underlying spatial representation. In other words, the underlying representation is heavily rooted in the director's perspective, and effort is required to infer the novel perspective. With this inferencing process as a backdrop, in the present study, perspective taking was used to tackle two primary goals: (1) to assess whether the choice of locatives might reflect something about the underlying representational structure and (2) to determine what effect, if any, describing visually experienced versus inferred perspectives has on the memory for spatial displays. Answering such questions required new stimuli and methods.
In the present experiment, the displays consisted of seven unique objects on a disk, and the director's task was to explain to the matcher how to reconstruct the display from a given orientation. This feature of the experiment allowed us to examine both the director's description of the display and the quality of the matcher's reconstruction based on that description. In addition, the small number of objects made it possible to test the director's memory for the display after he or she had described it verbally.

Because we were interested in the director's mental representation of the space both during and after the description, two important changes were made in the relationship between the director and the matcher. First, the director described the layout with limited feedback from the matcher. Previous work has indicated that the quality of the descriptions is dependent on having an actual listener (Schober, 1998), and pilot work indicated that directors made more errors in their descriptions when no listener was actually reconstructing the display. Therefore, rather than having no matcher, we tried to minimize the contributions of the matcher to the perspective-taking exercise. To accomplish this, the matcher was required to listen to the description and indicate when a given placement had been executed. Second, the matcher was unaware of the location of the director, relative to the display. This change was intended to effectively eliminate director-centered locatives in favor of a much stronger perspective-taking requirement.

Other important features of the experiment were the use of additional measures during the description of the display and the use a larger set of offset conditions $\left(0^{\circ}-180^{\circ}\right.$ in $45^{\circ}$ steps, both clockwise and counterclockwise). Finally, the director participated in spatial memory tasks following his or her description of the display. These memory tasks provided the critical measures for making inferences about the nature of memory representations acquired during the viewing and description of the display. By having both the description and the memory tasks, the nature of the memory representation could be compared with the ability to imagine and describe alternative perspectives.

If taking another observer's perspective requires creating a generalized representation of the space, we would expect to see evidence for this generalization at test. This generalization might be manifested as pure viewpointindependent performance - namely, equivalent performance for all the learned and novel viewpoints. It could also be reflected more subtly as a reduction in the cost for novel viewpoints when different perspectives were described (non- $0^{\circ}$ offsets), as compared with when the same perspective was described $\left(0^{\circ}\right.$ offset). For example, we might expect participants to perform better (more quickly and/or more accurately) with the view of the display they saw and/or the view they described than with novel views, but the cost for novel views might be larger for $0^{\circ}$ than for non- $0^{\circ}$ offsets. Alternatively, if taking novel perspectives does not involve a generalized representation, we would expect to see better performance on 
the visually perceived view and/or the view that the participants described, relative to novel views of the display. Moreover, the cost associated with novel views would be equivalent for $0^{\circ}$ and non- $0^{\circ}$ offset conditions.

\section{METHOD}

\section{Participants}

Eighty participants volunteered to participate in return for course credit in introductory psychology. Half of the participants ( 20 males and 20 females) participated as directors, and the remaining half (20 males and 20 females) participated as matchers. Director-matcher dyads consisted of 10 male-male pairs, 10 male-female pairs, 10 female-female pairs, and 10 female-male pairs. Pairs were randomly assigned to the experimental groups defined below, with equal numbers of each pair type in each group. This counterbalancing was done to avoid any effects due to possible gender differences.

\footnotetext{
Materials

Displays. An initial configuration of objects was selected from the two configurations used in previous studies of large-scale space (Shelton \& McNamara, 1997, 2001a). The configuration was centered on a 50 -cm-diameter felt-covered wood disk. Seven common objects were randomly assigned to the configuration five different times, creating five different displays (see Figure 2). For each dyad, one display was selected as the target display and used for the verbal description phase.

Offset conditions. Five groups were formed, corresponding to the offset conditions of $0^{\circ}, 45^{\circ}, 90^{\circ}, 135^{\circ}$, and $180^{\circ}$. Within the $45^{\circ}$, $90^{\circ}$, and $135^{\circ}$ groups, half were clockwise and half were counterclockwise positions. As is shown in Figure 3, the director's position was always labeled $0^{\circ}$ (for the sake of the memory tasks), and different matcher's positions were labeled according to the degree of offset from the director's position.

Set-up. The set-up involved a table set against the wall of a $12 \times$ $14 \mathrm{ft}$ room. The table was split with a divider to define the director's station (left half) and the matcher's station (right half). The director's station had the complete display and an arrow indicating the perspective to be described. The matcher's station had an empty disk and a set of objects to be placed. The matcher was not informed of the director's location relative to the display. A microphone and tape recorder were placed near the director to record the verbal description of the space.
}

Judgments of relative direction. For judgments of relative direction, trials were formed using two-object headings and a third object as the target (e.g., "Imagine you are at the car and facing the candle. Point to the battery"). Each two-object heading represented one of eight imagined headings ranging from $0^{\circ}$ to $315^{\circ}$ in $45^{\circ}$ increments. Trials were coded either clockwise for offsets to the left of the director or counterclockwise for offsets to the right of the director. Right and left were irrelevant for the $0^{\circ}$ and $180^{\circ}$ offsets, so half of the participants in each of these conditions were randomly assigned to either the right or the left group for coding the imagined headings.

Scene recognition. For scene recognition, the participants were required to discriminate pictures of the layout they had seen and described from pictures of the other four layouts (which they had neither seen nor described; see the Displays section, above). Digitized photographs of each of the five displays were taken from 24 orientations beginning at $0^{\circ}$ and continuing in $15^{\circ}$ increments around the entire display. Images from the target display were the old trials, whereas images from the remaining four displays were new trials. The orientations of the images were labeled according to the direction of the offset, as in judgments of relative direction.

\section{Procedure}

Descriptions. The participants were given instructions and placed at their respective stations. The director described the display to the matcher, and the matcher attempted to reconstruct the display. The verbal description was recorded, along with the time required by the matcher to complete the description. The matcher was instructed not to speak during the description. Instead, the matcher was given a clicker and was instructed to click once when a command was completed or to click twice to have a command repeated/clarified. Once the director finished describing the display, he or she indicated so by saying, "Done."

Reconstructions. During the description, the matcher tried to place the objects on the blank display on the basis of the director's instructions. If a command was completed, the matcher clicked one time. If the matcher wanted a command repeated, he or she clicked twice. When the director indicted that the description was finished, the matcher clicked one final time in agreement. The matcher's reconstruction was evaluated using bidimensional regression and an index of distortion (Waterman \& Gordon, 1984). The bidimensional regression provided a measure of rotation error indicating how close the reconstructed orientation was to the intended orientation (as defined by the offset condition). The distortion index (DI)

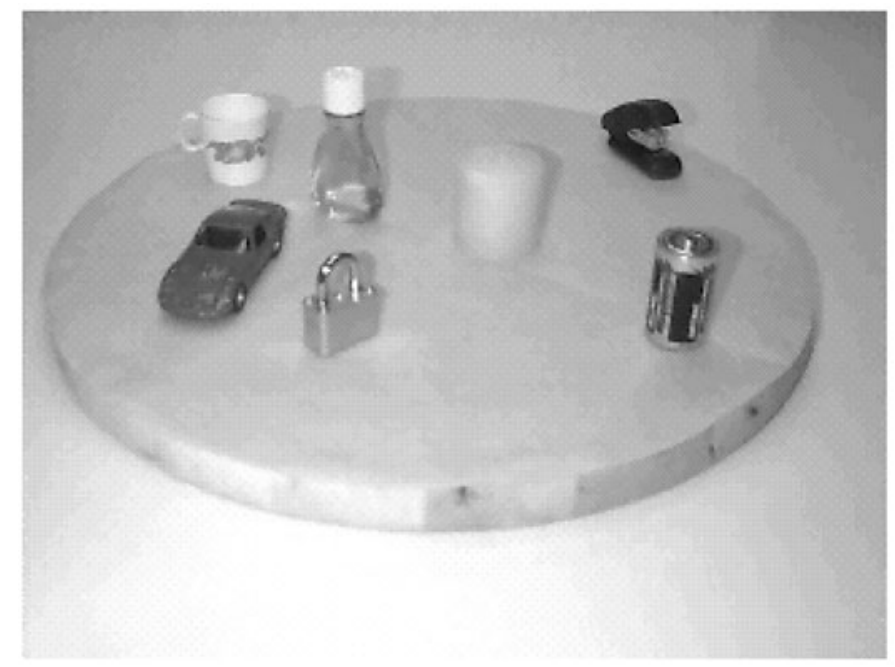

Figure 2. One of the layouts used in the experiment. 




Figure 3. Schematic showing the offset conditions. The viewing position for the director is designated as $0^{\circ}$. The remaining positions show clockwise and counterclockwise offsets.

converted the bidimensional regression coefficient into a measure of percentage of distortion that was corrected for scale, rotation, and translation, allowing assessment of the accuracy with which relative positions were reproduced.

Judgments of relative direction. Following the description, the director participated in the judgments of relative direction. Trials were presented individually on a Macintosh computer and proceeded as follows: (1) The participant clicked the mouse to begin a trial; (2) the trial statement (e.g., "Imagine you are at the car and facing the candle. Point to the battery") was displayed above a circle and a moveable line, creating a dial; (3) the participant then used the mouse to point the line on the circle in the direction of the target object and clicked the mouse. On each trial, the angular error and the response latency of the pointing judgment were measured.

Scene recognition. After judgments of relative direction, the director took a short break, then participated in the scene recognition task. Photographs were approximately $8.2 \mathrm{~cm}$ high $\times 11.2 \mathrm{~cm}$ wide and were presented individually in the center of a black screen on a Macintosh computer. Images remained on the screen until the participant responded with a keypress. After each keypress, the screen was blank for 1,000 msec before the next image appeared. The test consisted of 384 trials, presented in four blocks of 96 trials. Each block contained two instances of each of the 24 target trials and 48 distractor trials. Trials were randomized within each block, and the participants were allowed short breaks between blocks. The participants were instructed to respond by indicating whether the presented image came from the learned display or from one of the distractor displays by pressing the appropriate key. They were also informed that images were taken from several orientations. The participants were asked to respond quickly but accurately. Both accuracy and response latency were recorded.
Map drawing. After the experiment, the participants were given a blank sheet of paper and were asked to draw the display. The key information from the map drawings was the choice of orientation for the drawing.

\section{RESULTS}

\section{Descriptions}

The verbal descriptions provided by the director were coded for the total number of locatives, errors in locatives, and the types of locatives used. Number of locatives and errors were analyzed separately using between- subjects analyses of variance (ANOVAs) with degree of offset as a factor. No significant effects were observed.

The types of locatives were initially evaluated according to the classifications used by Schober $(1993,1995)$ : director centered (e.g., "The car is about two inches directly in front of me"), matcher centered (e.g., "Assuming you are at 6 o'clock, the battery is at about 3 o'clock"), both centered (only in the $0^{\circ}$ offset condition; e.g., "If the display is a compass, the bottle is between west and southwest as you look at the display"), object centered (e.g., "The lock is about 3 inches away from the car's right door"), and neutral (e.g., "The candle is at the very center of the circle"). After an examination of the frequency with which these types of locatives were used, we found that only three types of locatives were actually 
used: matcher centered (non- $0^{\circ}$ offsets), both centered $\left(0^{\circ}\right.$ offsets $),{ }^{1}$ and object centered. For the analysis, matcherand both-centered locatives were classified as orientation dependent and were compared with the objectcentered locatives. On the average, directors used more orientation-dependent locatives $(67 \%)$ than objectcentered locatives $[33 \% ; F(1,35)=47.86]$, and this effect did not differ across the offset conditions $(F<1)$. The participants consistently reported trying to describe the matcher's view, even in the $0^{\circ}$ offset condition, and this is revealed in the large percentage of orientationdependent locatives.

\section{Reconstructions}

Rotation errors and DIs were analyzed using betweensubjects ANOVAs with degree of offset and direction of offset as factors. The rotation errors were minimal $(M=$ $\left.5^{\circ}, S D=1.02\right)$, whereas the DIs were highly variable $(M=22, S D=10.3)$. There were no significant effects in either measure; however, there was a trend for the nonegocentric offsets to be more distorted (mean DI = 28 ) than the $0^{\circ}$ offset (mean DI $=19, p=0.20$ ).

\section{Judgments of Relative Direction}

Absolute angular error and response latency were analyzed separately using split-plot ANOVAs with offset condition, imagined heading, and pointing direction as factors. No significant effects were observed for response latency. For angular error, only significant effects and interactions are reported $(\alpha=0.01)$.

Figure 4 shows angular error as a function of imagined heading and offset condition, where the visually perceived view for all the groups was $0^{\circ}$ and the de- scribed view is labeled according to the degree of offset (i.e., $45^{\circ}$ for the $45^{\circ}$ offset condition). The striking result is that people tended to be most accurate on trials oriented with the described view, as compared with all the novel views, as supported by the main effect of imagined heading $[F(7,245)=16.42]$. Simple main effect contrasts also support this observation: For the $0^{\circ}$ offset, $0^{\circ}$ headings were most accurate $[F(1,245)=65.50]$; for the $45^{\circ}$ offset, $45^{\circ}$ headings were most accurate $[F(1,245)=$ 33.39]; for the $90^{\circ}$ offset, $90^{\circ}$ headings were most accurate $[F(1,245)=41.60]$; for the $135^{\circ}$ offset, $135^{\circ}$ headings were most accurate $[F(1,245)=23.85]$; and for the $180^{\circ}$ offset, $180^{\circ}$ headings were most accurate $[F(1,245)=13.21]$. In addition, the significant interaction between imagined heading and offset condition $[F(28,245)=7.12]$ appeared to be due to a benefit for the visually perceived view at $0^{\circ}$ in the non- $0^{\circ}$ offset conditions, such that $0^{\circ}$ headings were more accurate than all the novel headings for the $45^{\circ}$ offset $[F(1,245)=11.93]$, the $90^{\circ}$ offset $[F(1,245)=47.35]$, and the $180^{\circ}$ offset $[F(1,245)=16.06]$. Moreover, in the $90^{\circ}$ and $180^{\circ}$ offset conditions, $0^{\circ}$ headings did not differ from $90^{\circ}$ and $180^{\circ}$ headings, respectively $(F \mathrm{~s}<1)$. For the $45^{\circ}$ offset, $0^{\circ}$ headings were less accurate than $45^{\circ}$ headings $[F(1,245)=4.43]$, and for the $135^{\circ}$ offset, $0^{\circ}$ headings were less accurate than $135^{\circ}$ headings $[F(1,245)=6.71]$. These results, shown in Figure 5, suggest that both the visually perceived view at $0^{\circ}$ and the described view were mentally represented in the non- $0^{\circ}$ offset conditions.

In addition to effects of orientation, the main effect of pointing direction was significant $[F(5,175)=8.93]$. Pointing direction results were consistent with previous studies (see Shelton \& McNamara, 1997, 2001a) showing that accuracy is better for pointing to the front than for

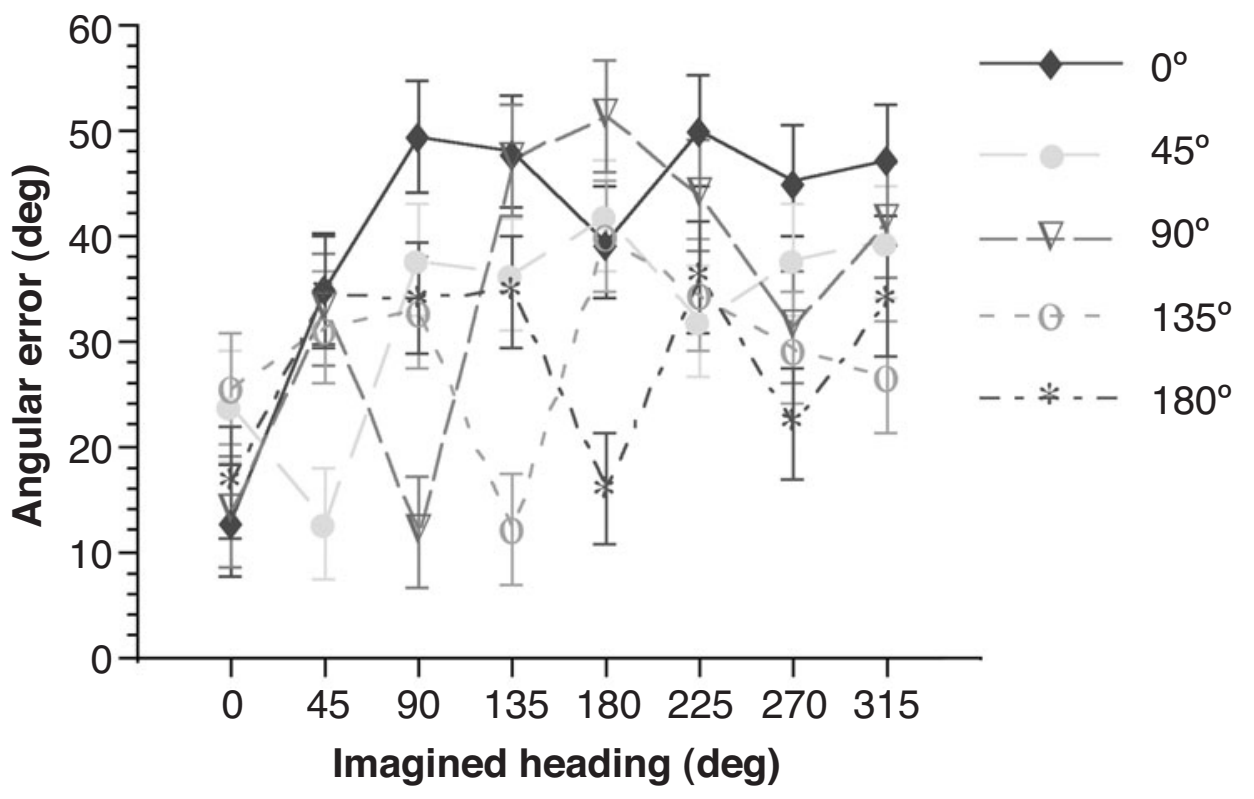

Figure 4. Mean angular error in judgments of relative direction as a function of imagined heading and offset condition. An error bar reflects \pm 1 standard error of the mean. 


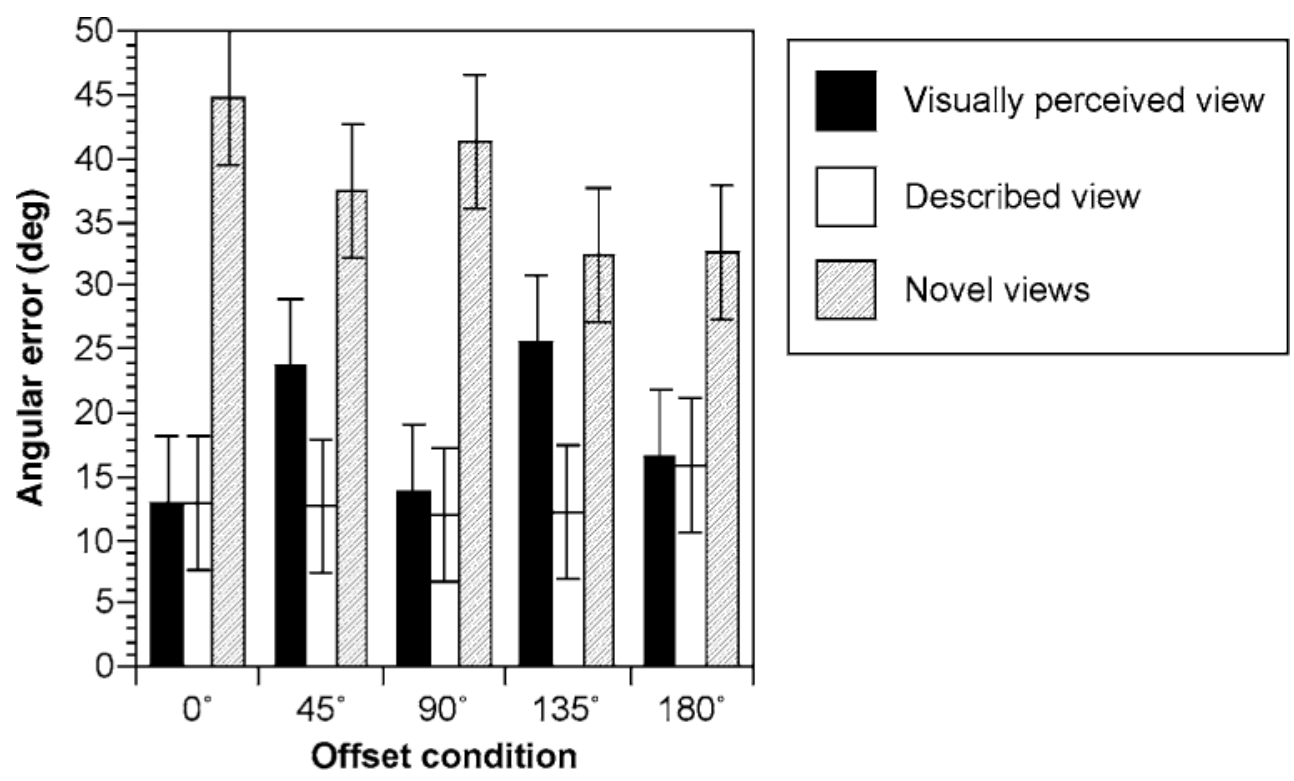

Figure 5. Mean angular error in judgments of relative direction as a function of offset condition for the visually perceived view, the described view, and the novel views. For the $0^{\circ}$ offset condition, the data for the visually perceived view and those for the described view are identical. An error bar reflects \pm 1 standard error of the mean.

pointing to the sides and better for the sides than for the back. Pointing direction also interacted with imagined headings $[F(35,1225)=2.49]$; however, the effect was small and appeared to be due to exaggerated effects when participants were pointing to the back. In any case, the main effect and interaction do not appear to compromise conclusions about imagined heading and offset condition.

\section{Scene Recognition}

The overall miss and false alarm rates were $2.5 \%$ and $1.8 \%$, respectively. Because error rates were so low, they were not analyzed further. Response latencies were analyzed using split-plot ANOVAs with offset condition and orientation as factors. The only reliable effect was the main effect of orientation $[F(23,805)=7.74]$. As is indicated in Figure 6, performance was fastest on trials oriented with $0^{\circ}[F(1,805)=141.99]$. This corresponds to the visually perceived view for all the participants. The interaction between orientation and offset condition was not significant $[F(92,805)=1.00]$. Figure 7 shows the means for the visually perceived view, the described view, and the novel views for each offset condition. Unlike judgments of relative direction, there was no benefit for the described view in scene recognition.

\section{Map Drawing}

Map accuracy was assessed using a DI based on bidimensional regression (Waterman \& Gordon, 1984). Maps were generally very accurate (mean DI $=7.2, S D=$ 1.34 ), and no differences were observed for different offset conditions $(F<1)$. The map-drawing data were also used as a gross measure of which view people preferred. The assumption was that they would choose to draw the map from the orientation that was most accessible. In the $0^{\circ}$ offset condition, all 8 participants drew the map from the $0^{\circ}$ orientation, as was expected. In the $45^{\circ}$ offset condition, all 8 participants drew maps from the $45^{\circ}$ orientation. Likewise, all 8 participants in the $90^{\circ}$ offset condition drew maps from the $90^{\circ}$ orientation. In the $135^{\circ}$ offset condition, 7 participants drew maps from the $135^{\circ}$ orientation, and 1 drew the map from the $0^{\circ}$ orientation. In the $180^{\circ}$ offset condition, 5 participants drew maps from the $180^{\circ}$ orientation, and 3 drew them from the $0^{\circ}$ orientation. As in judgments of relative direction, people appeared to prefer using the described view, suggesting that the same source of information might be useful for map drawing and judgments of relative direction.

\section{Test Order}

In this experiment, all the participants performed judgments of relative direction before scene recognition. To determine whether task order affected the pattern of results, five additional pairs (one per offset) were tested, with scene recognition first. The results were identical to those reported above.

\section{DISCUSSION}

We set out to answer questions about how spatial descriptions inform us about and participate in spatial memory. In terms of the use of different locatives, we did not replicate Schober's (1995) finding that people shift to 


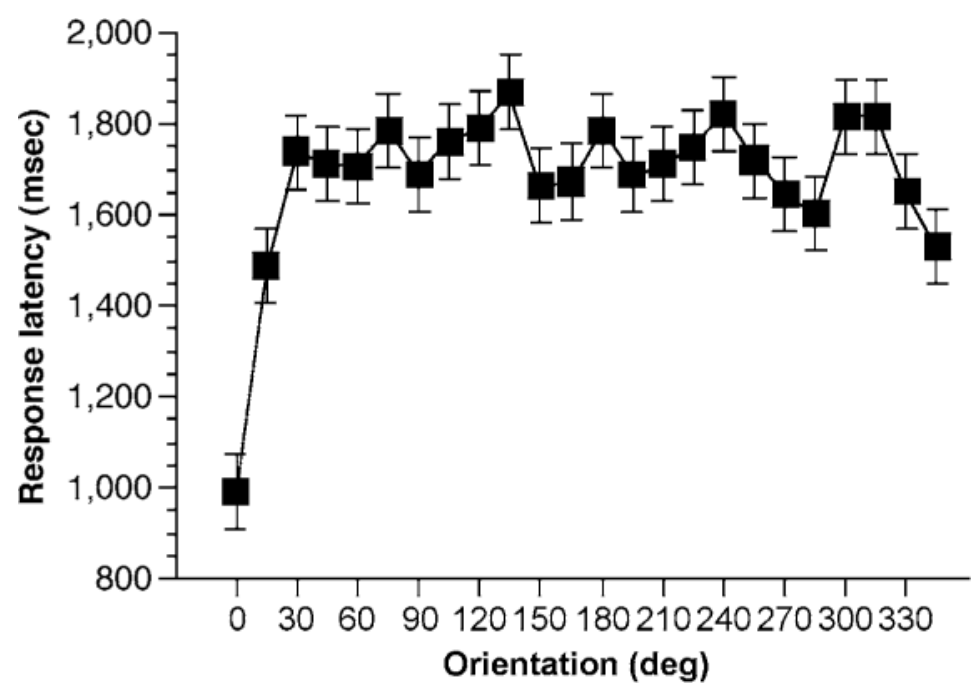

Figure 6. Mean response latencies for scene recognition as a function of orientation. Error bars reflect \pm 1 standard error of mean.

more neutral locatives when describing an orientation other than their own. Regardless of offset conditions, the participants opted to use the matcher's orientation (assuming another person's egocentric perspective). Several methodological differences between this experiment and Schober's (1995) experiments might have led to this disparity in results. First, the displays in the present experiment differed from Schober's (1995). Rather than having a set display for the matcher to work from, the director was required to help the matcher reconstruct the display on a blank disk. In addition, Schober's (1995) displays had many more individual items than did the displays in our experiment. These differences may have reduced the potential neutral locatives because there were fewer fixed items available for use as reference points. Notably, when object-centered locatives were used, they tended to appear later in the descriptions, supporting the contention that the present displays did not lend themselves to the more neutral locatives. Second, in Schober's (1995) experiments, directors and matchers were able to converse freely and could communicate about the reference systems that were being used. More recently, Schober (1998) has suggested that when matchers are not allowed to communicate, directors will work harder to minimize confusion. The use of matcher-centered locatives in our experiment may reflect this desire to make the matcher's job easier.

In addition to the locatives, we included measures of time and accuracy during the descriptions. These measures also failed to provide clear evidence of the ease with which nonegocentric perspectives were taken, as compared with egocentric perspectives. However, there was a trend for the descriptions to lead to poorer reconstructions in the nonegocentric conditions, suggesting that the non $-0^{\circ}$ offset descriptions may have been less precise. Unfortunately, the variability of the reconstructions made clear conclusions difficult.
A second major purpose of the experiment was to investigate how perspective taking (egocentric vs. nonegocentric) might affect the subsequent memory representation. With regard to this goal, there were two key findings. First, the results clearly indicated that even though people successfully described viewpoints other than their own during the description phase, the accuracy and speed of their memory for the space was still markedly dependent on the particular viewpoints experienced. These results suggest that a generalized representation of the layout was not stored either before or after the director gave a verbal description of the space. However, the specific conditions of the perspective-taking task did have an important effect on the subsequent memory. The views that were experienced (visually perceived and described) influenced what was ultimately accessible during the memory tests. Moreover, the results speak to how differences in the information available from each view interacted with the way in which memory was probed.

The primary result of interest was the viewpointdependent performance in both judgments of relative direction and scene recognition. In both cases, there was a preference for a familiar view (or views), as compared with all the novel views. In judgments of relative direction, both the visually perceived view and the described view appeared to have an advantage over the novel views, suggesting that both were accessible in memory. However, the degree to which they were accessible varied for different offset conditions. In the $90^{\circ}$ and $180^{\circ}$ offset conditions, the visually perceived view $\left(0^{\circ}\right)$ did not differ from the described view; thus, both were equally accessible. In the $45^{\circ}$ and $135^{\circ}$ offset conditions, the visually perceived view $\left(0^{\circ}\right)$ was imagined more accurately than the novel views but was not as accurately imagined as the described view. This was surprising given that the visually perceived view corresponded to something that the participants actually saw, whereas the 


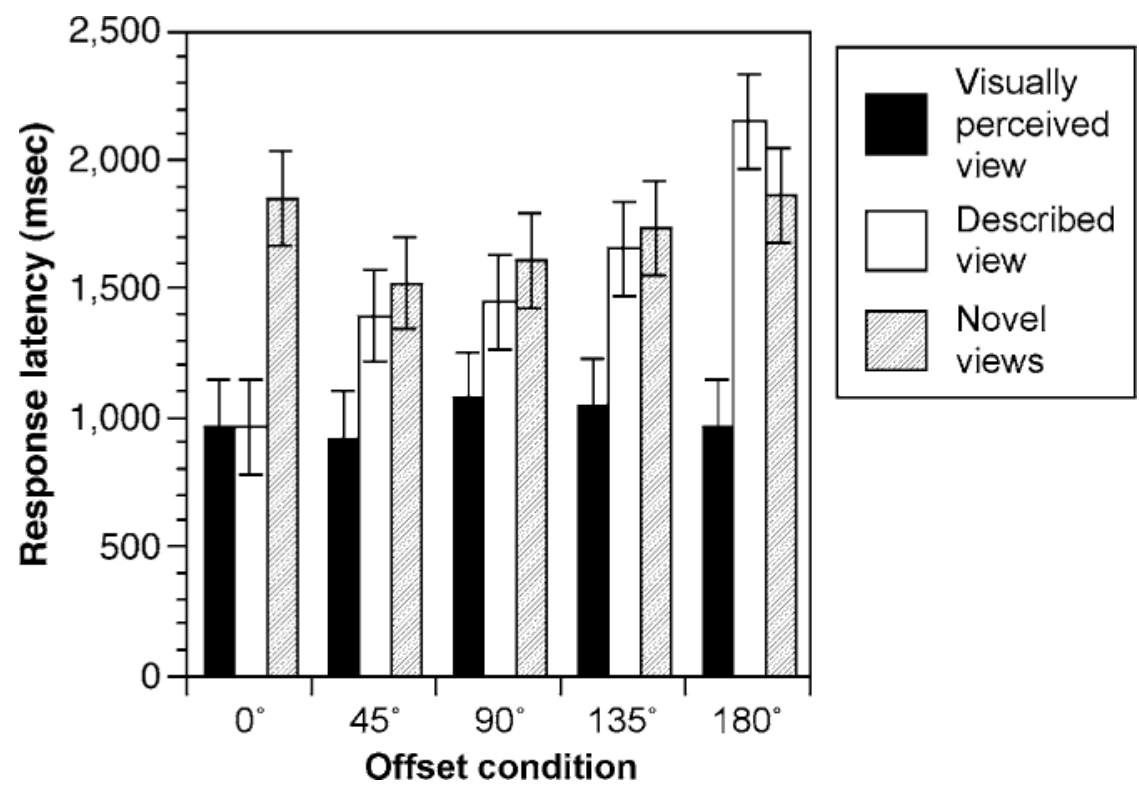

Figure 7. Mean response latency for scene recognition as a function of offset condition for the visually perceived view, the described view, and the novel views. For the $0^{\circ}$ offset condition, the data for the visually perceived view and those for the described view are identical. An error bar reflects \pm 1 standard error of mean.

described view was only "seen" to the extent that people used mental images in developing their description. This difference suggests that the information acquired from the described view was not necessarily visual but did provide sufficient information in memory for making judgments of relative direction. This same information also appeared to be useful for map drawing after the experiment, given that the majority of the participants chose the orientation of the described view for orienting their maps.

This lack of visual information in the described view is consistent with the scene recognition results. In scene recognition, performance was fastest at orientations corresponding to the visually perceived view $\left(0^{\circ}\right)$ for all offset conditions, and there was no apparent benefit for the described view. Scene recognition is a visual task, so it is not surprising that people could not recognize the "unseen" view as quickly as they could recognize the visually perceived view. It is, however, interesting that the described view, which clearly showed a benefit in judgments of relative direction, appeared to be no different from a novel view during scene recognition.

The differences in performance on the two memory tests suggest that people were storing information about both views (visually perceived and described) but that the degree to which they were able to access these different views depended directly on the type of information available for each view and the type of information required by the particular memory test. Visually perceiving a view provided information sufficient for improved performance, relative to novel views, in both judgments of relative direction and scene recognition. Describing a view (possibly with imagery) provided in- formation sufficient for making accurate judgments of relative direction (and in certain conditions, judgments based on information from the described view were more accurate than those based on information obtained from the visually perceived view), yet it was not sufficient for fast scene recognition. Visually recognizing the described view required just as much effort as recognizing novel views. Explanations for this task difference are discussed in the context of the following framework.

Collectively, the results from this experiment are consistent with a theoretical framework proposed by Shelton and McNamara (2001a; see also Mou \& McNamara, 2002). According to this theory, learning the spatial structure of a new environment involves interpreting it in terms of a spatial reference system. This process is analogous to determining the "top" of a figure; in effect, conceptual "north" is assigned to the layout, creating privileged directions in the environment (conceptual "north" need not, and usually will not, correspond to true or magnetic north or any other cardinal direction). Our present hypothesis is that the spatial structure of the environment is represented in terms of an intrinsic reference system (e.g., Palmer, 1989), one defined by the layout itself (e.g., the rows and columns formed by chairs in a classroom). This intrinsic reference system is selected on the basis of egocentric experience, spatial and nonspatial properties of the objects, and even instructions. A collection of objects will have an infinite number of possible intrinsic axes, but because of perceptual grouping principles, such as proximity and similarity, some of these will be more salient than others. Egocentric experience will also make some axes more salient than others. 
For example, in one of the experiments reported by Mou and McNamara (2002), objects were placed on a square mat oriented with the walls of the surrounding room (see Figure 8; real objects were used, not words). Participants studied the layout from $315^{\circ}$ and were instructed to learn the layout along the egocentric $315^{\circ}$ axis or the nonegocentric $0^{\circ}$ axis. This instructional manipulation was accomplished by pointing out that the layout could be seen in "columns" consistent with the appropriate axis and by asking the participants to point to the objects in the appropriate order when they were quizzed during the learning phase (e.g., clock, jar, scissors, shoe, etc. vs. scissors, clock, wood, shoe, jar, etc.). All the participants viewed the layout from $315^{\circ}$. After learning, the participants made judgments of relative direction, using their memory of the layout. The most important finding was that the participants who were instructed to learn the layout along the nonegocentric $0^{\circ}$ axis were more accurate pointing to objects from the $0^{\circ}$ heading than from the $315^{\circ}$ heading (which is the view they actually saw), whereas the participants who were instructed to learn the layout along the egocentric $315^{\circ}$ axis were more accurate pointing to objects from the $315^{\circ}$ heading than from the $0^{\circ}$ heading. The overall error was equivalent in the two groups, indicating that there was no cost to learning the layout from along a nonegocentric axis.

In the present experiment, the participants viewed the layout from one perspective and described it from another (the visually perceived and described views were identical in the $0^{\circ}$ offset condition). We believe that each of these two activities resulted in the selection of an intrinsic reference axis for representing the locations of objects; speaking metaphorically, the visually perceived view established conceptual "north" $\left(0^{\circ}\right)$, whereas the described view established (working counterclockwise) conceptual "northwest" $\left(45^{\circ}\right)$, "west" $\left(90^{\circ}\right)$, or "south-

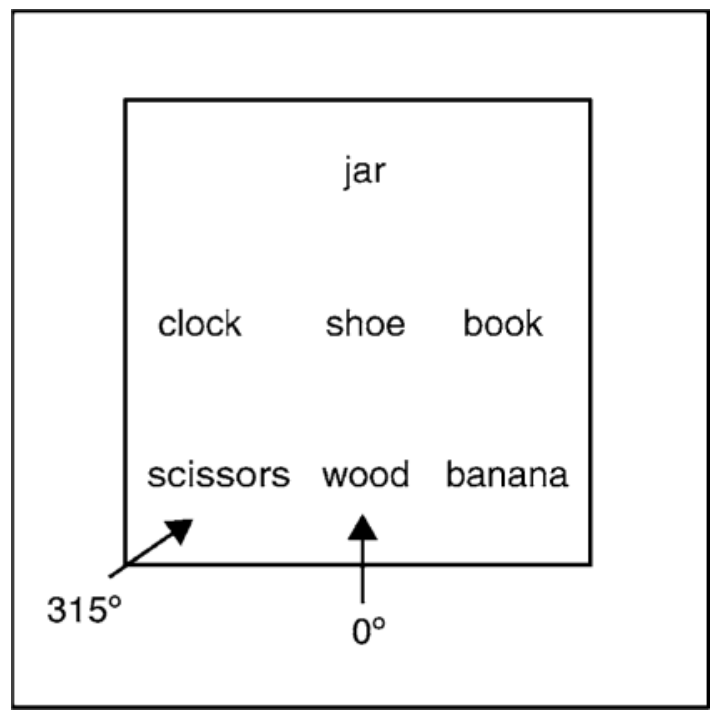

Figure 8. Illustration of the layout used by Mou and McNamara (2002). west" $\left(135^{\circ}\right)$. The relatively poor performance in judgments of relative direction on the $0^{\circ}$ heading for the participants who described the layout from $45^{\circ}$ and from $135^{\circ}$ (see Figure 5) may indicate that people have difficulty representing spatial layouts in terms of oblique axes $\left(0^{\circ}\right.$ and $45^{\circ}$ or $0^{\circ}$ and $\left.135^{\circ}\right)$. The dominance of the described view in these conditions may reflect the fact that it was the primary focus of the learning phase.

Why, according to this explanation, is scene recognition sensitive only to the visually perceived view? One possible explanation is that a single representation of the layout was formed at the time it was described and this representation contained information about the spatial relations among objects, as specified from the visually perceived and the described perspectives, and a visual memory of what the layout looked like from the visually perceived view (but not from the described view). Another possible explanation is that two representations were formed: One preserved interobject spatial relations, whereas the other preserved visual appearance (for an elaboration, see McNamara, 2003; Shelton \& McNamara, 2004). It is not obvious to us that deciding between these alternatives is important (or even possible). The important conclusion that we reach is that the dissociation between the two tasks indicates that the two tasks are sensitive to different kinds of spatial information.

These task difference may reflect a type of encoding specificity (e.g., Thomson \& Tulving, 1970) that can arise from having different representations (or components) of the same spatial layout. Scene recognition requires a visual match; thus, it is logical that having the direct visual perception of a view might facilitate retrieval of that view from memory. For judgments of relative direction, the task itself does not necessarily require direct visual information. Instead, it requires knowledge of relative locations (distances and directions). This information is available (or at least derivable) through direct visual perception, but it is essentially required for describing the locations of objects. Different experiences (in this case, visual perception and verbal description) of the same space can, therefore, lead to qualitative differences in the nature of the information represented. Although these representations provide some common information about spatial layout, they have distinguishable qualities based on the way in which the information was experienced. The visually perceived view and the described view clearly afforded different information when it came time to retrieve spatial layout information, suggesting that the visual and the nonvisual experiences were not equivalent in terms of how spatial information was represented.

One potentially important factor that we did not control in this experiment was the "amount" of visual versus descriptive experience the participants had. Given that the description was occurring simultaneously with the viewing, it would be difficult to equate such things. Although it is possible that we would see different nuances in the data if we were to impose controls for time or effort on task, the data clearly suggest that neither experi- 
ence (visual or descriptive) was entirely better or worse than the other. Instead, it was the case that the two experiences afforded different information.

In this study, we introduced only one type of nonvisual experience - the experience associated with providing a verbal description- because describing scenes and giving/receiving directions are familiar activities for most people. However, at an even simpler level, our experiences with space may also involve a rich tapestry of sensory experiences, including tactile, proprioceptive, and auditory cues to location. For example, in a related study, we demonstrated robust representations of space after manual reconstruction (Shelton \& McNamara, 2001b). The study was conducted much like the present experiment, except that the participants viewed a spatial display from one orientation and manually reconstructed it without vision from another perspective. The results revealed a vivid representation of the manually reconstructed view, emphasizing the multimodal nature of spatial memory. Unlike the described view in the present study, the manually reconstructed view was preferred in both judgments of relative direction and scene recognition, suggesting that the nonvisual experience was actually dominating both the visual representation and the representation of spatial relations. This difference raises important questions about differences among the different nonvisual experiences we might have, suggesting that some are more integrated and confusable with visual experiences than are others. For example, all the participants in the manual reconstruction experiment reported using mental imagery to aid in reconstructing the display, whereas 28 of the 40 directors in the present experiment reported the use of imagery. This difference might indicate a more central role for imagery in reconstruction, as compared with verbal descriptions, allowing this nonvisual view to dominate all aspects of the spatial representation. Further investigations into the differences among modalities will help clarify these differences in results.

A great deal of research from nonhuman primates has suggested that space is represented in multiple modalities, perhaps even in separate representations (e.g., Andersen, Snyder, Bradley, \& Xing, 1997; Colby \& Goldberg, 1999; Graziano, Yap, \& Gross, 1994). Combined with previous studies, the present work suggests that our understanding of the representations arising from different modalities and experiences depends on establishing a more detailed account of what different experiences entail perceptually and cognitively. In addition to addressing the nature of these experiences, understanding these differences requires careful consideration of the tasks we use to explore memory for spatial layout.

\section{REFERENCES}

Andersen, R. A., Snyder, L. H., Bradley, D. C., \& Xing, J. (1997). Multimodal representation of space in the posterior parietal cortex and its use in planning movements. Annual Review of Neuroscience, 20, 303-330.

CLARK, H. H., \& WiLKES-GibBs, D. (1986). Referring as a collaborative process. Cognition, 22, 1-39.
Colby, C. L., \& Goldberg, M. E. (1999). Space and attention in parietal cortex. Annual Review of Neuroscience, 22, 319-349.

EHRICH, V., \& KosTER, C. (1983). Discourse organization and sentence form: The structure of room descriptions in Dutch. Discourse Processes, 6, 169-195.

GARROD, $\bar{S} .$, \& ANDERSON, A. (1987). Saying what you mean in dialogue: A study in conceptual and semantic coordination. $\underline{\text { Cognition, }}$ 27, 181-218.

GraZIANO, M. S. A., Yap, G. S., \& Gross, C. G. (1994). Coding of visual space by premotor neurons. Science, 266, 1054-1057.

IsAACS, E. A., \& CLARK, H. H. (1987). References in conversation between experts and novices. Journal of Experimental Psychology: General, 116, 26-37.

LEVINSON, S. C. (1996). Frames of reference and Molyneux's question: Crosslinguistic evidence. In P. Bloom, M. A. Peterson, L. Nadel, \& M. F. Garrett (Eds.), Language and space (pp. 109-169). Cambridge, MA: MIT Press.

Linde, C., \& LABov, W. (1975). Spatial structure as a site for the study of language and thought. Language, 51, 924-939.

McNamara, T. P. (2003). How are the locations of objects in the environment represented in memory? In C. Freksa, W. Brauer, C. Habel, \& K. F. Wender (Eds.), Spatial cognition III: Routes and navigation, human memory and learning, spatial representation and spatial reasoning. (Lecture Notes in Artificial Intelligence, Vol. 2685, pp. 174191). Berlin: Springer-Verlag.

Mou, W., \& McNamara, T. P. (2002). Intrinsic frames of reference in spatial memory. Journal of Experimental Psychology: Learning, Memory, \& Cognition, 28, 162-170.

PALMER, S. E. (1989). Reference frames in the perception of shape and orientation. In B. E. Shipp \& S. Ballesteros (Eds.), Object perception: Structure and process (pp. 121-163). Hillsdale, NJ: Erlbaum.

ScHOBER, M. F. (1993). Spatial perspective-taking in conversation. Cognition, 47, 1-24.

SCHOBER, M. F. (1995). Speakers, addressees, and frames of reference: Whose effort is minimized in conversations about locations. Discourse Processes, 20, 219-247.

SCHOBER, M. F. (1998). How addressees affect spatial perspective choice in dialogue. In P. Olivier \& K.-P. Gapp (Eds.), Representation and processing of spatial expressions (pp. 231-245). Mahwah, NJ: Erlbaum.

Shelton, A. L., \& McNamara, T. P. (1997). Multiple views of spatial memory. Psychonomic Bulletin \& Review, 4, 102-106.

Shelton, A. L., \& MCNAmARA, T. P. (2001a). Systems of spatial reference in human memory. Cognitive Psychology, 43, 274-310.

Shelton, A. L., \& McNamara, T. P. (2001b). Visual memories from nonvisual experiences. Psychological Science, 12, 343-347.

Shelton, A. L., \& McNamara, T. P. (2004). Orientation and perspective dependence in route and survey learning. Journal of Experimental Psychology: Learning, Memory, \& Cognition, 30, 158-170.

TAYLOR, H. A., \& TVERSKY, B. (1992). Spatial mental models derived from survey and route descriptions. Journal of Memory \& Language, 31, 261-292.

TAYLOR, H. A., \& Tversky, B. (1996). Perspective in spatial descriptions. Journal of Memory \& Language, 35, 371-391.

Thomson, D. M., \& Tulving, E. (1970). Associative encoding and retrieval: Weak and strong cues. Journal of Experimental Psychology, 86, 255-262.

Waterman, S., \& Gordon, D. (1984). A quantitative-comparative approach to analysis of distortion in mental maps. Professional Geographer, 36, 326-337.

\section{NOTE}

1. One director-centered locative was used by a director in the $0^{\circ}$ offset condition. For the sake of the analysis, this was treated as both centered.

(Manuscript received January 21, 2003; revision accepted for publication October 31, 2003.) 